\title{
Prevention of lumbar disc degeneration through co- manipulation of insulin-like growth factor 1 and vascular endothelial growth factor
}

\section{Shan Zhao}

Fudan University

\section{Zuo-Zhou Ye}

Fudan University

Zuo-qing Liu ( $\nabla$ cxr67981@yeah.net)

Fudan University https://orcid.org/0000-0003-1908-9175

\section{Research}

Keywords: Lumbar disc degeneration (LDD), gene therapy, insulin-like growth factor 1 (IGF1), vascular endothelial growth factor (VEGF), adeno-associated virus (AAV)

Posted Date: August 12th, 2020

DOI: https://doi.org/10.21203/rs.3.rs-54211/v1

License: (c) (i) This work is licensed under a Creative Commons Attribution 4.0 International License. Read Full License 


\section{Abstract \\ Background:}

Associated with abnormal angiogenesis and disc dysfunction, Lumbar disc degeneration (LDD) appears to be an important disease suffered by aged people. Previous studies have highlighted the importance of insufficient insulin-like growth factor 1 (IGF1) and excessive vascular endothelial growth factor (VEGF) in the development and progression of LDD, whereas a practical therapeutic strategy is lacking.

\section{Results:}

Here, we did a gene therapy approach using adeno-associated virus (AAV) carrying both IGF1 and shVEGF (AAV-IGF1/shVEGF), which simultaneously corrected IGF1 and VEGF issues in a rat model for LDD.We found that AAV-IGF1/shVEGF significantly reduced disc cell death in the vertebral pulp and annulus fibrosus and significantly improved the lumbar proteoglycan and collagen II levels. Therefore, this approach effectively prevented the development of LDD.

\section{Conclusions:}

Our study presents a novel therapeutic approach on LDD, which is clinically translatable.

\section{Background}

Many aged people suffer from potent low back pain from lumbar disc degeneration (LDD) [1-3]. The lumbar disc has several components, including nucleus pulposus (NP), annulus fibrosus and cartilage end plates. The primary pathogenesis of LDD results from excessive cartilage-specific extracellular matrix production by apoptotic NP cells [4]. Therefore, the current therapeutic strategy treating LDD is focusing on reduction of apoptosis of NP cells [5-9].

Many factors play roles in the pathogenesis of LDD. The insulin-like growth factors (IGFs) have been shown to antagonize progression of LDD, by us [10-12] and by others [13-15]. Specifically, we have shown that IGF1 induced phosphorylation of receptor of IGF1 (IGF1R), leading to phosphorylation of its downstream factor Akt and subsequently nuclear exclusion of FoxO1, to eventually suppress matrix metalloproteinase 3 (MMP3)-mediated LDD [10]. On the other hand, abnormally activation of angiogenesis has also been shown to promote progression of LDD [16-19]. For example, degenerative intervertebral disc disorders have been associated with increased expression of vascular endothelial growth factor (VEGF), the most potent angiogenic factor [17]. VEGF family has 6 members, among which VEGF-A is most important one and is referred as VEGF in this article. Although these previous studies have highlighted the importance of IGF1 and VEGF in the development and progression of LDD, whereas a practical therapeutic strategy is lacking. 
Here, we reported a gene therapy approach using adeno-associated virus (AAV) carrying IGF1 and shVEGF (AAV-IGF1/shVEGF), which corrected both IGF1 and VEGF issues in a rat model for LDD. We found that AAV-IGF1/shVEGF significantly reduced disc cell death in the vertebral pulp and annulus fibrosus and significantly improved the lumbar proteoglycan and collagen II levels. Thus, our study presents a novel therapeutic approach on LDD, which is clinically translatable.

\section{Results}

\section{Bioinformatics analyses on IGF1 and VEGF levels in LDD patients}

Since previous studies have highlighted the importance of IGF1 and VEGF in the development and progression of LDD, we first analyzed the levels of IGF1 and VEGF in disc specimens from normal control and LDD patients. We performed GEO database mining and reanalyzed the data from public database. The Limma R package was used to identify genes that were significantly altered in LDD, compared to normal controls. Shown in a Volcano map (Fig. 1A), we found that IGF1 was significantly low expressed in LDD, while VEGF was significantly high expressed in LDD, compared to normal controls (Fig. 1B). Thus, these data indicate that downregulation of IGF1 and upregulation of VEGF may both contribute to LDD development and suggest that reversal of these features could attenuate or even inhibit the process.

\section{Preparation Of Aavs Overexpressing Igf1 And Depleting Vegf}

In order to figure out whether our hypothesis is correct, we thus used an AAV technique to enhance IGF1 signaling and suppress VEGF-mediated angiogenesis in one construct. The experimental AAV used an AAV serotype 2 carrying recombinant IGF1 and shVEGF under a CMV promoter, while 2 respective scramble controls were used in the control AAV (Fig. 2A). We transduced a disc cell line, HNPSV, with the two AAVs and compared to untreated cells. We found that AAV-IGF1/shVEGF-transduced cells expressed significantly higher levels of IGF1 than controls, by mRNA (Fig. 2B) and by ELISA for protein (Fig. 2C). Moreover, AAV-IGF1/shVEGF-transduced cells expressed significantly lower levels of VEGF than controls, by mRNA (Fig. 2D) and by ELISA for protein (Fig. 2E). Thus, the quality of the AAVs was validated.

\section{Aav-igf1/shvegf Treatment Increases Igf1 In Spine Tissue After Ldd}

Next, we used in a rat model of LDD to examine the effects of this gene therapy by AAV-IGF1/shVEGF. Forty rats were randomly divided into 4 groups of 10. Group 1: sham. Group 2: LDD and an orthotopic saline injection. Group 3: LDD and an orthotopic AAV-scr injection. Group 4: LDD and an orthotopic AAVIGF1/shVEGF injection. Rats were kept for 8 weeks before analysis. First, we examined the levels of IGF1 
in the rat vertebral pulp and annulus fibrosus by immunohistochemistry (Fig. 3A) and by ELISA (Fig. 3B). We found that the IGF1 signaling was modestly increased in group 2 and 3, compared to group 1, while was increased much more in group 4 (Fig. 3A-B). Moreover, the number of nucleus pulposus cells in group 2 and 3 appeared to reduce, compared to group 1. But this number improved in group 4 (Fig. 3A). Together, these data confirm that AAV-IGF1/shVEGF treatment increases IGF1 in spine tissue after LDD.

\section{Aav-igf1/shvegf Treatment Decreases Vegf In Spine Tissue After Ldd}

Next, we examined the levels of VEGF in the rat vertebral pulp and annulus fibrosus by immunohistochemistry (Fig. 4A) and by ELISA (Fig. 4B). We found that the VEGF signaling was dramatically and significantly increased in group 2 and 3 , compared to group 1 , while this increase in VEGF was significantly attenuated in group 4 (Fig. 4A-B). Thus, these data confirm that AAVIGF1/shVEGF treatment decreases VEGF in spine tissue after LDD.

\section{AAV-IGF1/shVEGF treatment reduces cell apoptosis in spine tissue after LDD}

After validation of the AAV-IGF1/shVEGF treatment, we examined the effect of AAV-IGF1/shVEGF treatment on LDD. The disc cell apoptosis is the most important feature of LDD, thus the cells from the rat vertebral pulp and annulus fibrosus were dissociated into single cell population for a flow-cytometrybased apoptosis assay. We found that the apoptotic cells were dramatically and significantly increased in group 2 and 3, compared to group 1, while this increase in apoptotic cells was significantly attenuated in group 4 (Fig. 5A-B). Thus, these data suggest that AAV-IGF1/shVEGF treatment reduces cell apoptosis in spine tissue after LDD.

\section{AAV-IGF1/shVEGF treatment enhances spine proteoglycan and collagen II after LDD}

The proteoglycan levels in spine decrease with the progression of LDD and faithfully represent the disease severity [20]. Moreover, levels of collagen II similarly decrease during LDD [21]. We thus examined the levels of spine proteoglycan and collagen II after treatments. Our data showed that the levels of proteoglycan and collagen II were dramatically and significantly decreased in group 2 and 3 , compared to group 1, while this decrease in proteoglycan and collagen II levels was significantly attenuated in group 4 (Fig. 6A-B). These data suggest that AAV-IGF1/shVEGF treatment enhances spine proteoglycan and collagen II after LDD. Thus, AAV-IGF1/shVEGF treatment significantly antagonizes development of LDD.

\section{Discussion}


Many factors and signaling pathways contribute to development of LDD. Among these factors and pathways, IGF1 signaling and VEGF pathway appeared to play critical roles. Interference with IGF1/IGFR signaling or VEGF pathway alone may not exert optimal effects on the prevention of LDD, since their downstream signaling cascades share some identical factors and could affect each other [22-26]. For example, we have shown that IGF1 induced phosphorylation of IGF1R, leading to phosphorylation of its downstream factor Akt and subsequently nuclear exclusion of FoxO1, to eventually suppress matrix metalloproteinase 3 (MMP3)-mediated LDD [10]. On the other hand, VEGF has been shown to signal partially through Akt/mTor signaling [27]. Hence, a co-manipulation of both factors may be more effective for reaching therapeutic need. Indeed, a previous study has targeted both IGF1 and VEGF to treat rodent model of amyotrophic lateral sclerosis (ALS), a fatal neurodegenerative disease characterized by motor neuron cell death in the cortex, brainstem, and spinal cord [28]. Therefore, in this study, we generated AAV vector to specifically targets both factors and evaluated its effects on LDD.

After in vitro and in vivo validation of the viral vectors, we showed that gene therapy co-addressing insulin-like growth factor 1 and vascular endothelial growth factor inhibits LDD progression and severity. Both decreases in proteoglycan and collagen II that occur in disc matrix molecules are known to contribute to loss of disc function with ageing and disc degeneration [29,30]. Moreover, the loss of biosynthetic capability of disc cells is very important for the pathogenesis of LDD, and could be the important targets for a successful therapy $[29,30]$. Here, our intervention significantly attenuated changes in these molecules, and thus provided a further promise in translating it into treatment for patients.

\section{Conclusion}

To the best of our knowledge, this strategy using orthotopic AAV-based gene therapy targeting both IGF1 pathway and angiogenesis is the first approach that successfully and effectively prevent LDD which is readily translatable to clinic. AAVs are now used in clinical trials and this orthotopic injection could be easily done in human patients. Hence, here we showed a novel strategy that could prevent LDD progression in a highly translatable way.

\section{Materials And Methods:}

\section{Protocol approval and animal treatments}

The present study was approved by the Research and Animal Ethics Association of Zhongshan Hospital affiliated to Fudan University (NO: GDR452). A total of 40 one-year-old female Sprague-Dawley rats (14$\sim 160 \mathrm{~g}$ ) were purchased from the Animal Laboratory of the Academy of Medical Sciences (Beijing, China) and used in strict accordance with the guidelines for the Care and Use of Laboratory Animals. The rats were kept in separate cages with free access to food and water, and in a twelve/twelve-hour light/dark cycle (temperature, $24^{\circ} \mathrm{C}$; humidity, 50\%). Rats were randomly divided into 4 groups of 10 . The sham group received only a skin incision, which was then sutured to close. The rats in the other 3 groups received surgical development of LDD, in which the sacrospinal muscles, spinous processes, 
supraspinous ligaments, interspinous ligaments and posterolateral halves of the bilateral zygapophysial joints of the lumbar spine were removed. Afterwards, the rats in LDD group received an orthotopic

injection of $150 \mu \mathrm{l}$ normal saline; rats in the LDD + AAV-scr group received an orthotopic injection of $10^{11}$ AAV-scr in $150 \mu$ l volume; rats in the LDD + AAV-IGF1/VEGF group received an orthotopic injection of $10^{11}$ AAV-IGF1/VEGF in $150 \mu$ volume. Rats were kept for 8 weeks before analysis.

\section{Cell Culture And Aav Preparation}

A human disc cell line, nucleus pulposus SV40 (HNPSV) has been described before [31]. The HNPSV cells were cultured in Dulbecco's modified Eagle medium (DMEM, Gibco; Life Technologies, Carlsbad, CA, USA) with $10 \%$ fetal bovine serum (FBS, Sigma-Aldrich, St Louis, MO, USA), penicillin $(100 \mu \mathrm{g} / \mathrm{ml})$ and streptomycin $(250 \mathrm{ng} / \mathrm{ml})$ at $37^{\circ} \mathrm{C}$, in a $5 \% \mathrm{CO}_{2}$ atmosphere. AAV serotype 2 vectors were generated by transfection of human embryonic kidney 293 cells. Rat IGF1 was prepared by PCR using CDNA from primary rat hepatic cells. Respective scramble sequence was used as control for rat IGF1. The sequence for shVEGF is 5'-TGTGAATGCAGACCAAAGA-3', and for its scramble is 5'-GGTATCTACTAGATGTACT-3' [32]. Transfection was performed with Lipofectamine 3000 reagent (Invitrogen, CA, Carlsbad, USA), according to the instructions of the manufacturer. The prepared virus was stored at $-80^{\circ} \mathrm{C}$. Titration of viral vectors was determined using a dot-blot assay.

\section{Sampling And Analysis By Immunohistochemistry And Elisa}

After euthanized by an intraperitoneal overdose of pentobarbital sodium, the lumbar spines of the rats, including the L4 to L6 discs, were removed. The vertebral pulp and annulus fibrosus were isolated for immunohistochemical and ELISA analysis. For immunohistochemistry, the metaphysis of the vertebral pulp and annulus fibrosus specimens were fixed in a $4 \%$ paraformaldehyde for 2 hours, then paraffinembedded followed by being cut into 5 - $\mu$ m-thick sections. The immunohistochemistry followed an ABC method, while DBA was used to develop IGF1 signals and fast red was used to develop VEGF signals. Primary antibodies are mouse anti-IGF1 (ab176523; Abcam, Cambridge, MA, USA; dilution: 1:300) and rabbit anti-VEGF (ab52917; Abcam; dilution: 1:800). Secondary antibodies are HRP-conjugated antimouse and anti-rabbit (Jackson ImmunoResearch Labs, West Grove, PA, USA). ELISA was performed using IGF1 kit (MG100; R\&D System, Los Angeles, CA, USA), VEGF kit (RRV00; R\&D System), proteoglycan kit (EKC39620; Biomatik, Wilmington, DE, USA) and collagen II kit (LS-F23885; LSBio, Seattle, WA, USA).

\section{Apoptosis Assay}

Cells were labeled with annexin V-FITC and propidium iodide (PI), using an apoptosis detecting kit (KeyGEN Biotech, Nanjing, China), and analyzed by flow cytometry using CellQuest software (BectonDickinson Biosciences, San Jose, CA, USA). 


\section{Rna Isolation, Quantitative Polymerase Chain Reaction (rt- qpcr)}

RNA extraction and cDNA synthesis were routine performed. RT-qPCR primers were: Rat GAPDH forward: 5'-TGATTCTACCCACGGCAAGTT-3', Rat GAPDH reverse: 5'-TGATGGGTTTCCCATTGATGA-3'; Rat VEGF forward: 5'-CAAGCCAAGGCGGTGAGCCA-3', Rat VEGF reverse: 5'-TCTGCCGGAGTCTCGCCCTC-3'; Rat IGF1 forward: 5'-TCTTGAAGGTGAAGATGCACACCA-3', Rat IGF1 reverse: 5'-CCTGAGGTTCTTCACAG-3'. Values were normalized against GAPDH, which proved to be stable across the samples, and then compared to experimental controls.

\section{Statistical Analysis And Bioinformatics}

All statistical analyses were carried out using the SPSS 20.0 statistical software package. Data were investigated using one-way ANOVA with a Bonferroni correction, followed by Fisher's exact test to compare 2 sub-groups. All values are shown as mean \pm standard deviation (SD) and are considered significant if $p<0.05$, not significant (NS) if $p>0.05$. For bioinformatics analysis, transcriptome RNAsequencing (RNA-seq) data of human disc specimens from normal and LDD patients were downloaded from the GEO data portal (https://www.ncbi.nlm.nih.gov/geo/). RNA-seq data of specimens in published database (GSE124272) were used for analysis by the R software Linear Models for Microarray and RNASeq Data (Limma) package. Pairwise differential expression was quantified using Cuffdiff. Cufflinks was used to determine FPKM levels for each gene from the STAR alignment and was used as input for Cuffdiff. Next, we performed differential gene analysis of all transcriptional data, setting a log2 Ifold change| $>1$ and a false discovery rate $(F D R)<0.05$ as the cutoff values.

\section{Declarations}

Ethics approval and consent to participate: The present study was approved by the Research and Animal Ethics Association of Zhongshan Hospital affiliated to Fudan University (NO: GDR452).

Consent for publication: not applicable

Availability of data and material: all included in the manuscript

Competing interests: The authors have declared that no competing interests exist.

Funding: Internal research funding

Authors' contributions: SZ, ZY and ZL conceived and designed the study. SZ, ZY and ZL performed the experiment. ZL wrote the manuscript. SZ and $Z Y$ helped to proofread the manuscript. All authors read and approved the final manuscript. 


\section{References}

1. Wang SZ, Rui YF, Lu J, Wang C. Cell and molecular biology of intervertebral disc degeneration: current understanding and implications for potential therapeutic strategies. Cell Prolif. 2014;47(5):381-90.

2. Wang HQ, Samartzis D. Clarifying the nomenclature of intervertebral disc degeneration and displacement: from bench to bedside. Int J Clin Exp Pathol. 2014;7(4):1293-8.

3. Risbud MV, Shapiro IM. Role of cytokines in intervertebral disc degeneration: pain and disc content. Nat Rev Rheumatol. 2014;10(1):44-56.

4. Feng $\mathrm{C}$, Liu H, Yang Y, Huang B, Zhou Y. Growth and differentiation factor-5 contributes to the structural and functional maintenance of the intervertebral disc. Cell Physiol Biochem. 2015;35(1):116.

5. Gruber HE, Ingram JA, Norton HJ, Hanley EN. Jr. Senescence in cells of the aging and degenerating intervertebral disc: immunolocalization of senescence-associated beta-galactosidase in human and sand rat discs. Spine. 2007;32(3):321-7.

6. Gruber HE, Norton HJ, Hanley EN Jr. Anti-apoptotic effects of IGF-1 and PDGF on human intervertebral disc cells in vitro. Spine. 2000;25(17):2153-7.

7. Gruber HE, Hanley EN Jr. Analysis of aging and degeneration of the human intervertebral disc. Comparison of surgical specimens with normal controls. Spine. 1998;23(7):751-7.

8. Wang L, Yang JK, Kabaleeswaran V, Rice AJ, Cruz AC, Park AY, Yin Q, Damko E, Jang SB, Raunser S, Robinson CV, Siegel RM, Walz T, et al. The Fas-FADD death domain complex structure reveals the basis of DISC assembly and disease mutations. Nat Struct Mol Biol. 2010;17(11):1324-9.

9. Park JB, Chang H, Kim KW. Expression of Fas ligand and apoptosis of disc cells in herniated lumbar disc tissue. Spine. 2001;26(6):618-21.

10. Liu Z, Zhou K, Fu W, Zhang H. Insulin-Like Growth Factor 1 Activates PI3k/Akt Signaling to Antagonize Lumbar Disc Degeneration. Cell Physiol Biochem. 2015;37(1):225-32.

11. Liu ZQ, Zhao S, Fu WQ. Insulin-like growth factor 1 antagonizes lumbar disc degeneration through enhanced autophagy. American journal of translational research. 2016;8(10):4346-53.

12. Liu ZQ, Fu WQ, Zhao S, Zhao X. Regulation of insulin-like growth factor 1 receptor signaling by microRNA-4458 in the development of lumbar disc degeneration. American journal of translational research. 2016;8(5):2309-16.

13. Li B, Zheng XF, Ni BB, Yang YH, Jiang SD, Lu H, Jiang LS. Reduced expression of insulin-like growth factor 1 receptor leads to accelerated intervertebral disc degeneration in mice. Int $\mathrm{J}$ Immunopathol Pharmacol. 2013;26(2):337-47.

14. Chen Z, Zhang W, Zhang N, Zhou Y, Hu G, Xue M, Liu J, Li Y. Down-regulation of insulin-like growth factor binding protein 5 is involved in intervertebral disc degeneration via the ERK signalling pathway. J Cell Mol Med. 2019;23(9):6368-77. 
15. Urano T, Narusawa K, Shiraki M, Usui T, Sasaki N, Hosoi T, Ouchi Y, Nakamura T, Inoue S. Association of a single nucleotide polymorphism in the insulin-like growth factor-1 receptor gene with spinal disc degeneration in postmenopausal Japanese women. Spine. 2008;33(11):1256-61.

16. David G, Ciurea AV, lencean SM, Mohan A. Angiogenesis in the degeneration of the lumbar intervertebral disc. Journal of medicine life. 2010;3(2):154-61.

17. Lee JM, Song JY, Baek M, Jung HY, Kang H, Han IB, Kwon YD, Shin DE. Interleukin-1 beta induces angiogenesis and innervation in human intervertebral disc degeneration. $\mathrm{J}$ Orthop Res. 2011;29(2):265-9.

18. Ali R, Le Maitre CL, Richardson SM, Hoyland JA, Freemont AJ. Connective tissue growth factor expression in human intervertebral disc: implications for angiogenesis in intervertebral disc degeneration. Biotechnic histochemistry: official publication of the Biological Stain Commission. 2008;83(5):239-45.

19. Wang K, Liu W, Song Y, Wu X, Zhang Y, Li S, Gao Y, Tu J, Liu Y, Yang C. The role of angiopoietin-2 in nucleus pulposus cells during human intervertebral disc degeneration. Lab Invest. 2017;97(8):97182.

20. Lipson SJ, Muir H. Experimental intervertebral disc degeneration: morphologic and proteoglycan changes over time. Arthritis Rheum. 1981;24(1):12-21.

21. Garnero P, Sornay-Rendu E, Arlot M, Christiansen C, Delmas PD. Association between spine disc degeneration and type II collagen degradation in postmenopausal women: the OFELY study. Arthritis Rheum. 2004;50(10):3137-44.

22. Dicarlo M, Bianchi N, Ferretti C, Orciani M, Di Primio R, Mattioli-Belmonte M. Evidence Supporting a Paracrine Effect of IGF-1/VEGF on Human Mesenchymal Stromal Cell Commitment. Cells Tissues Organs. 2016;201(5):333-41.

23. Maass A, Duzel S, Brigadski T, Goerke M, Becke A, Sobieray U, Neumann K, Lovden M, Lindenberger U, Backman L, Braun-Dullaeus R, Ahrens D, Heinze HJ, et al. Relationships of peripheral IGF-1, VEGF and BDNF levels to exercise-related changes in memory, hippocampal perfusion and volumes in older adults. Neuroimage. 2016;131:142-54.

24. Cao H, Jin C, Huang D, Liu C, Sun D, Tan C, Zhu X, Fei Y. Changes in serum IGF-1 level and tumor VEGF expression in mice with colorectal cancer under hyperglycemic conditions. Mol Med Rep. 2013;7(4):1361-5.

25. Sall JW, Klisovic DD, O'Dorisio MS, Katz SE. Somatostatin inhibits IGF-1 mediated induction of VEGF in human retinal pigment epithelial cells. Exp Eye Res. 2004;79(4):465-76.

26. Leske DA, Wu J, Fautsch MP, Karger RA, Berdahl JP, Lanier WL, Holmes JM. The role of VEGF and IGF-1 in a hypercarbic oxygen-induced retinopathy rat model of ROP. Mol Vis. 2004;10:43-50.

27. Trinh XB, Tjalma WA, Vermeulen PB, Van den Eynden G, Van der Auwera I, Van Laere SJ, Helleman J, Berns EM, Dirix LY, van Dam PA. The VEGF pathway and the AKT/mTOR/p70S6K1 signalling pathway in human epithelial ovarian cancer. Br J Cancer. 2009;100(6):971-8. 
28. Dodge JC, Treleaven CM, Fidler JA, Hester M, Haidet A, Handy C, Rao M, Eagle A, Matthews JC, Taksir TV, Cheng SH. Shihabuddin LS and Kaspar BK. AAV4-mediated expression of IGF-1 and VEGF within cellular components of the ventricular system improves survival outcome in familial ALS mice. Mol Ther. 2010;18(12):2075-84.

29. Wu B, Meng C, Wang H, Jia C, Zhao Y. Changes of proteoglycan and collagen II of the adjacent intervertebral disc in the cervical instability models. Biomed Pharmacother. 2016;84:754-8.

30. Scott JE, Haigh M. Proteoglycan-collagen interactions in intervertebral disc. A chondroitin sulphate proteoglycan associates with collagen fibrils in rabbit annulus fibrosus at the d-e bands. Biosci Rep. 1986;6(10):879-88.

31. Sakai D, Mochida J, Yamamoto Y, Toh E, Iwashina T, Miyazaki T, Inokuchi S, Ando K, Hotta T. Immortalization of human nucleus pulposus cells by a recombinant SV40 adenovirus vector: establishment of a novel cell line for the study of human nucleus pulposus cells. Spine. 2004;29(14):1515-23.

32. Xiao X, Prasadan K, Guo P, El-Gohary Y, Fischbach S, Wiersch J, Gaffar I, Shiota C, Gittes GK. Pancreatic duct cells as a source of VEGF in mice. Diabetologia. 2014;57(5):991-1000.

\section{Figures}


A
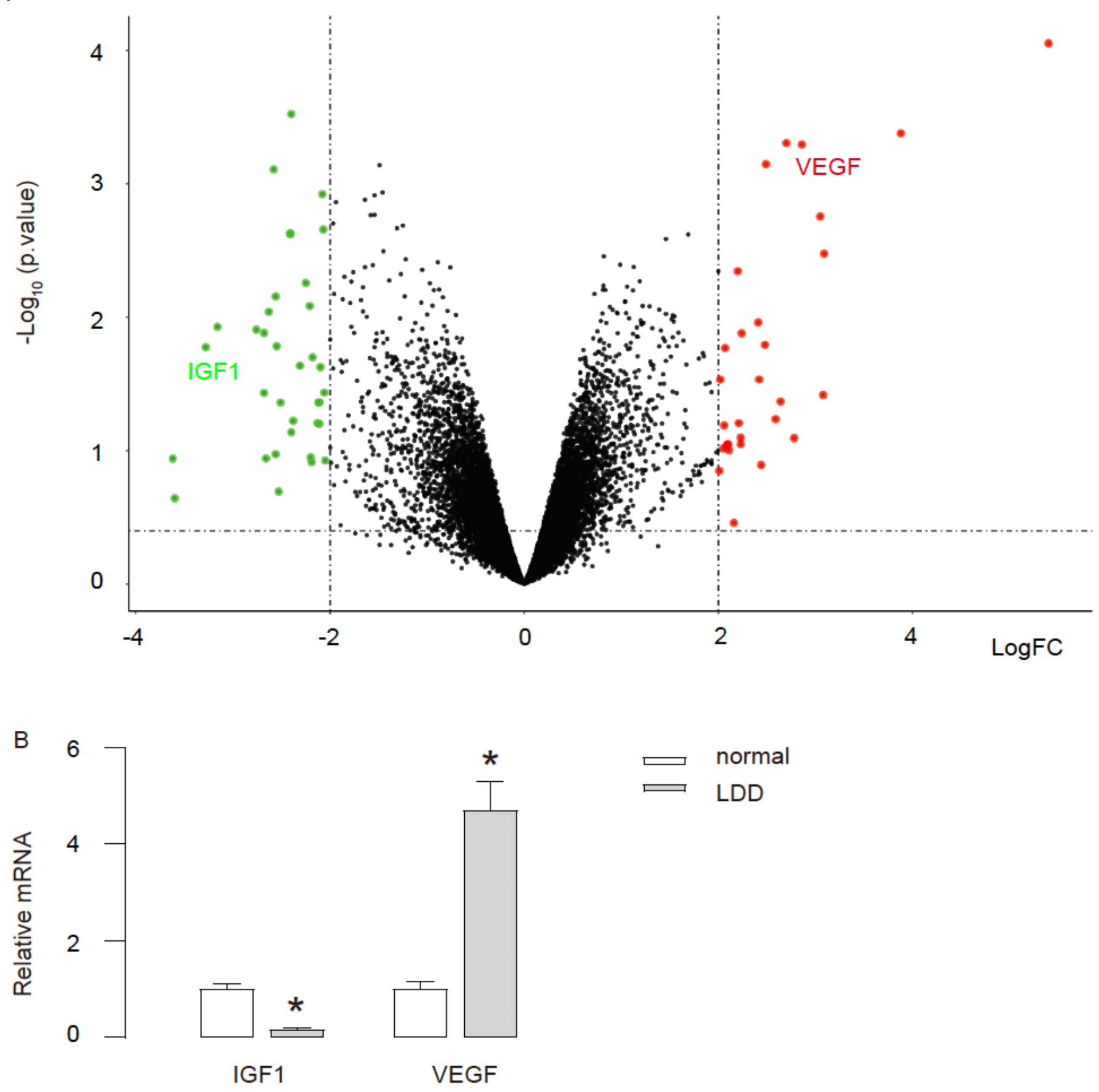

Figure 1

Bioinformatics analyses on IGF1 and VEGF levels in LDD patients (A) Volcano image for GEO database GSE124272) to compare gene profiling of disc cells in LDD patients to normal controls. Data were analyzed by R language. (B) Levels of IGF1 and VEGF in disc cells in LDD patients to normal controls. ${ }^{*} \mathrm{p}<0.05 . \mathrm{N}=16$. 
A
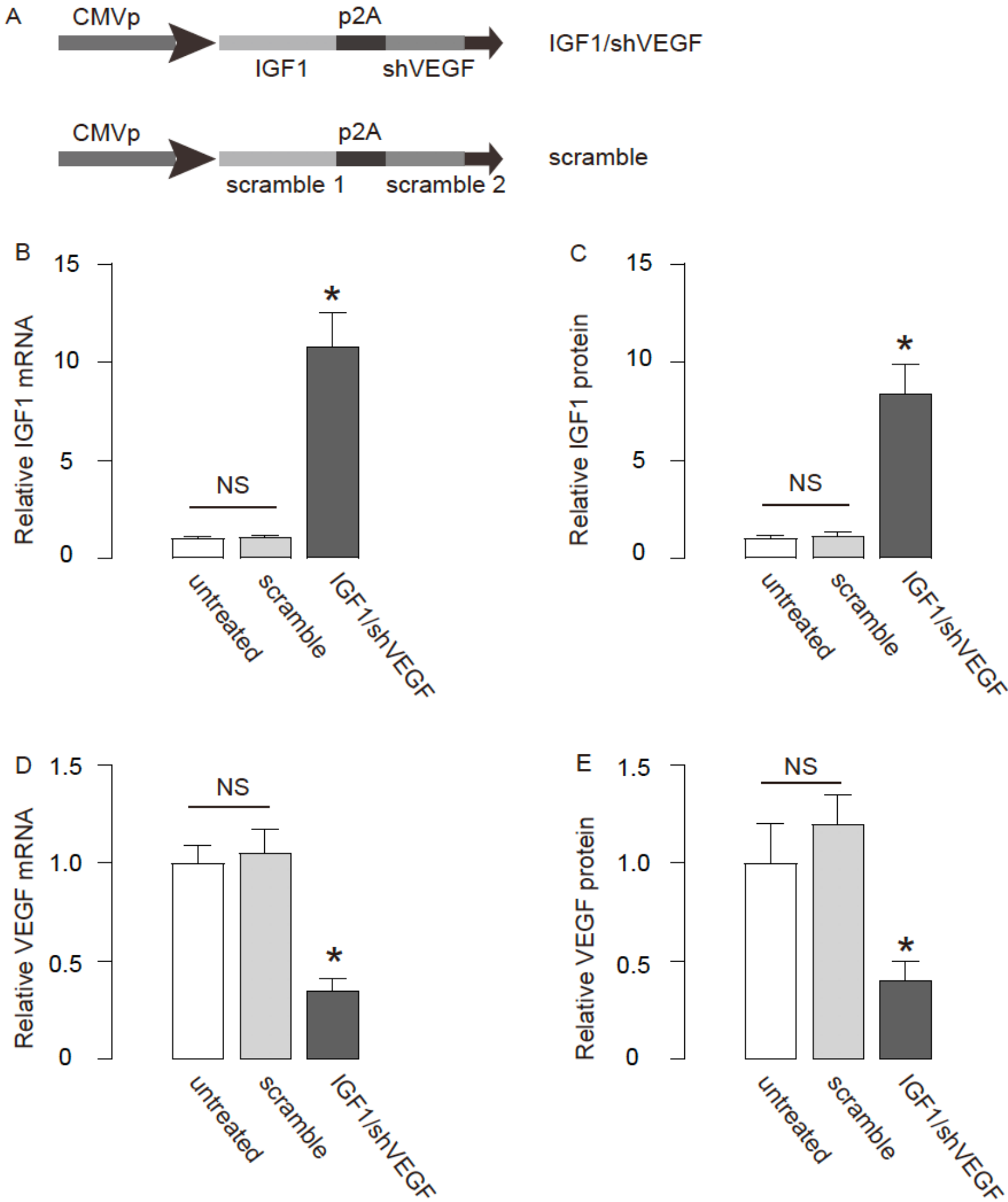

Figure 2

Preparation of AAVs overexpressing IGF1 and depleting VEGF (A) schematic to show structure of AAVIGF1/shVEGF and AAV-scramble viruses. The experimental AAV used an AAV serotype 2 carrying recombinant IGF1 and shVEGF (connected by a p2A structure) under a CMV promoter, while 2 respective scramble controls were used in the control AAV-scramble. (B-E) A disc cell line, HNPSV, was transduced 
with the two AAVs and compared to untreated cells. (B-C) IGF1 levels by RT-qPCR (B) and by ELISA (C). (D-E) VEGF levels by RT-qPCR (D) and by ELISA (E). * $p<0.05$. NS: non-significant. $N=5$.

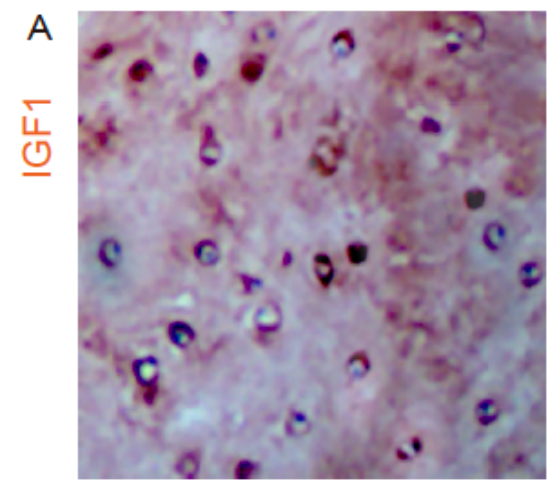

sham

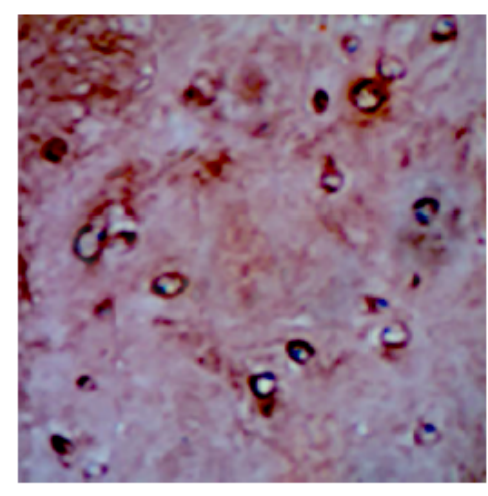

LDD+AAV-scr

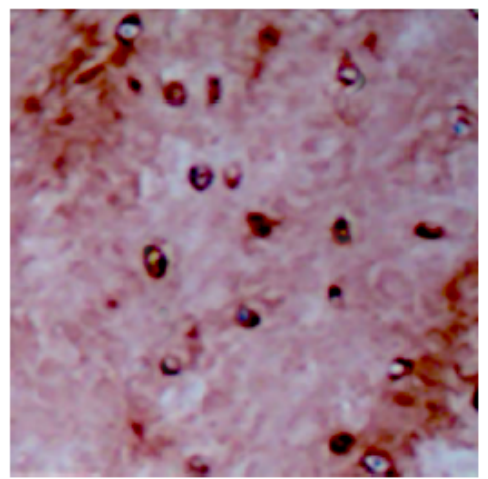

LDD

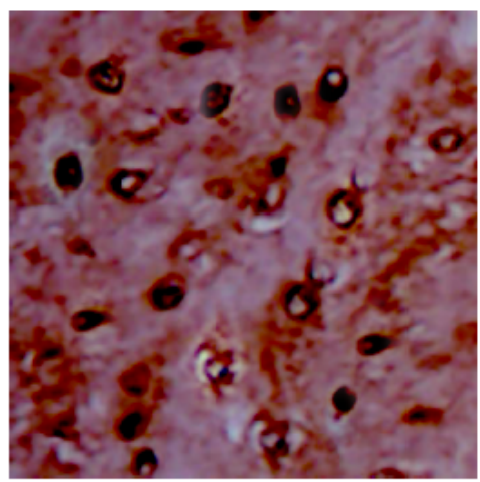

LDD+AAV-IGF1/shVEGF
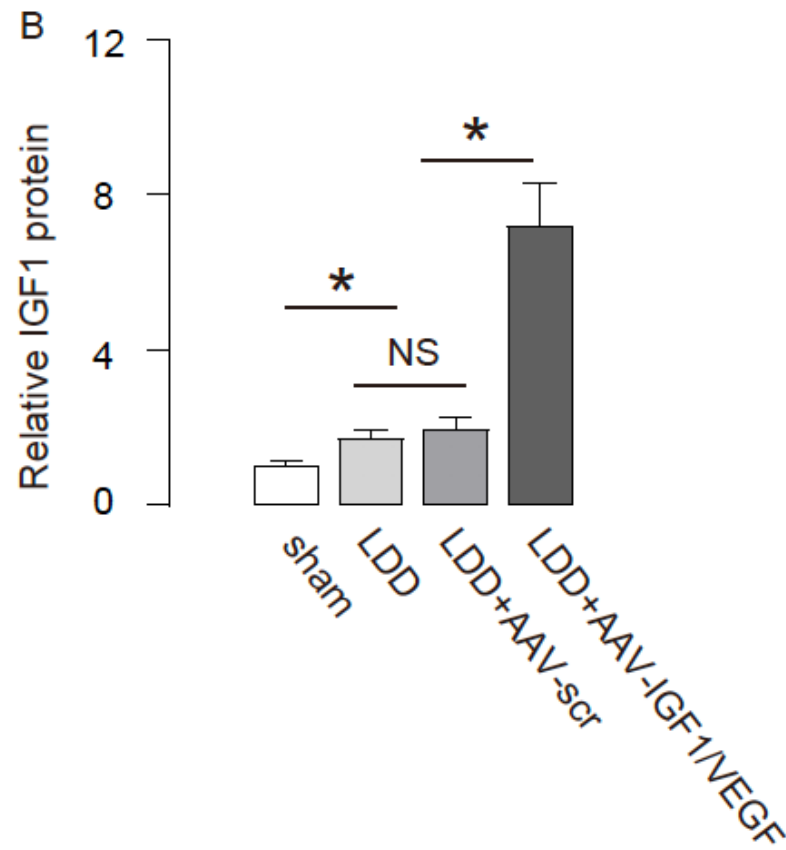

\section{Figure 3}

AAV-IGF1/shVEGF treatment increases IGF1 in spine tissue after LDD We used in a rat model of LDD to examine the effects of this gene therapy by AAV-IGF1/shVEGF. Forty rats were randomly divided into 4 groups of 10. Group 1: sham. Group 2: LDD and an orthotopic saline injection. Group 3: LDD and an orthotopic AAV-scr injection. Group 4: LDD and an orthotopic AAV-IGF1/shVEGF injection. Rats were kept for 8 weeks before analysis. (A-B) The levels of IGF1 in the rat vertebral pulp and annulus fibrosus 8 weeks after treatments were examined by immunohistochemistry $(A)$ and by ELISA (B). ${ }^{*} p<0.05$. NS: nonsignificant. $\mathrm{N}=10$. Scale bars are $20 \mu \mathrm{m}$. 


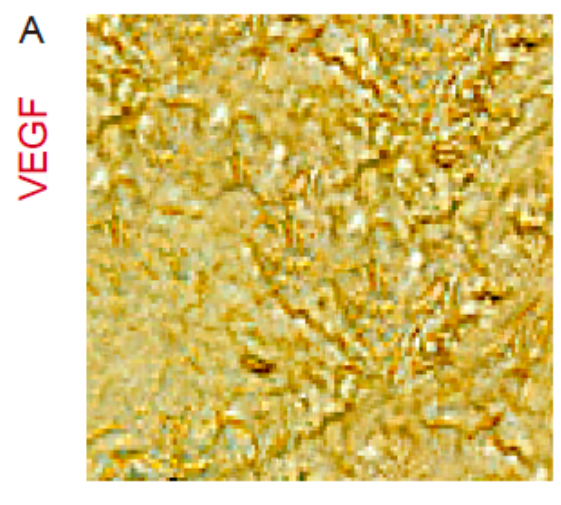

sham

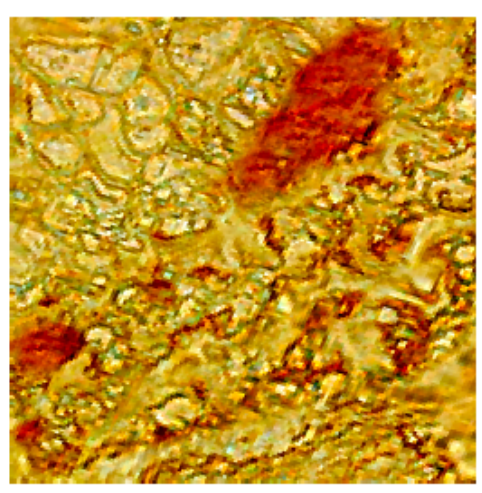

LDD+AAV-scr

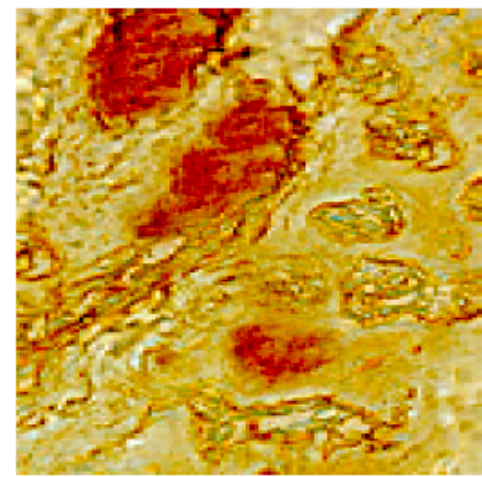

LDD

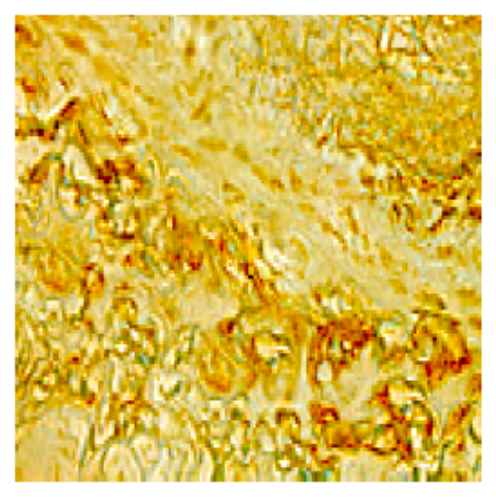

LDD+AAV-IGF1/shVEGF

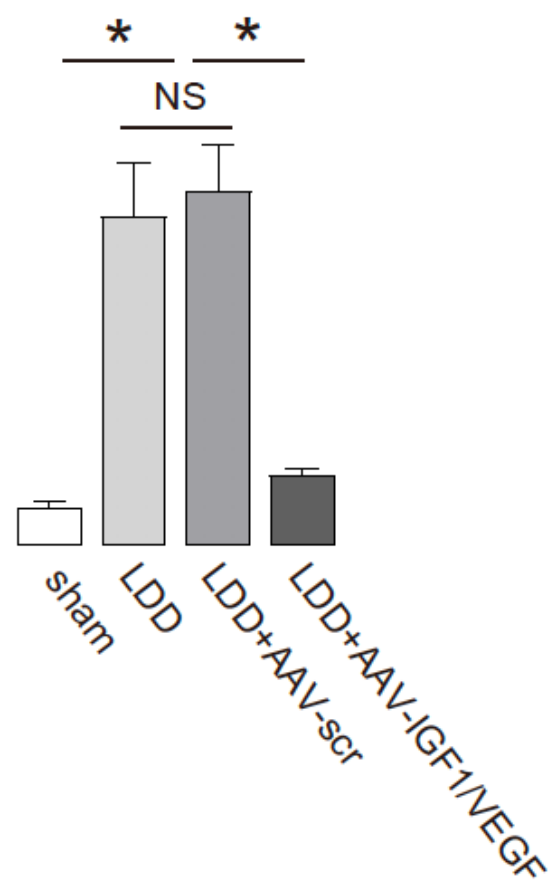

\section{Figure 4}

AAV-IGF1/shVEGF treatment decreases VEGF in spine tissue after LDD (A-B) The levels of VEGF in the rat vertebral pulp and annulus fibrosus 8 weeks after treatments were examined by immunohistochemistry (A) and by ELISA (B). ${ }^{*}<<0.05$. NS: non-significant. $N=10$. Scale bars are $20 \mu \mathrm{m}$. 

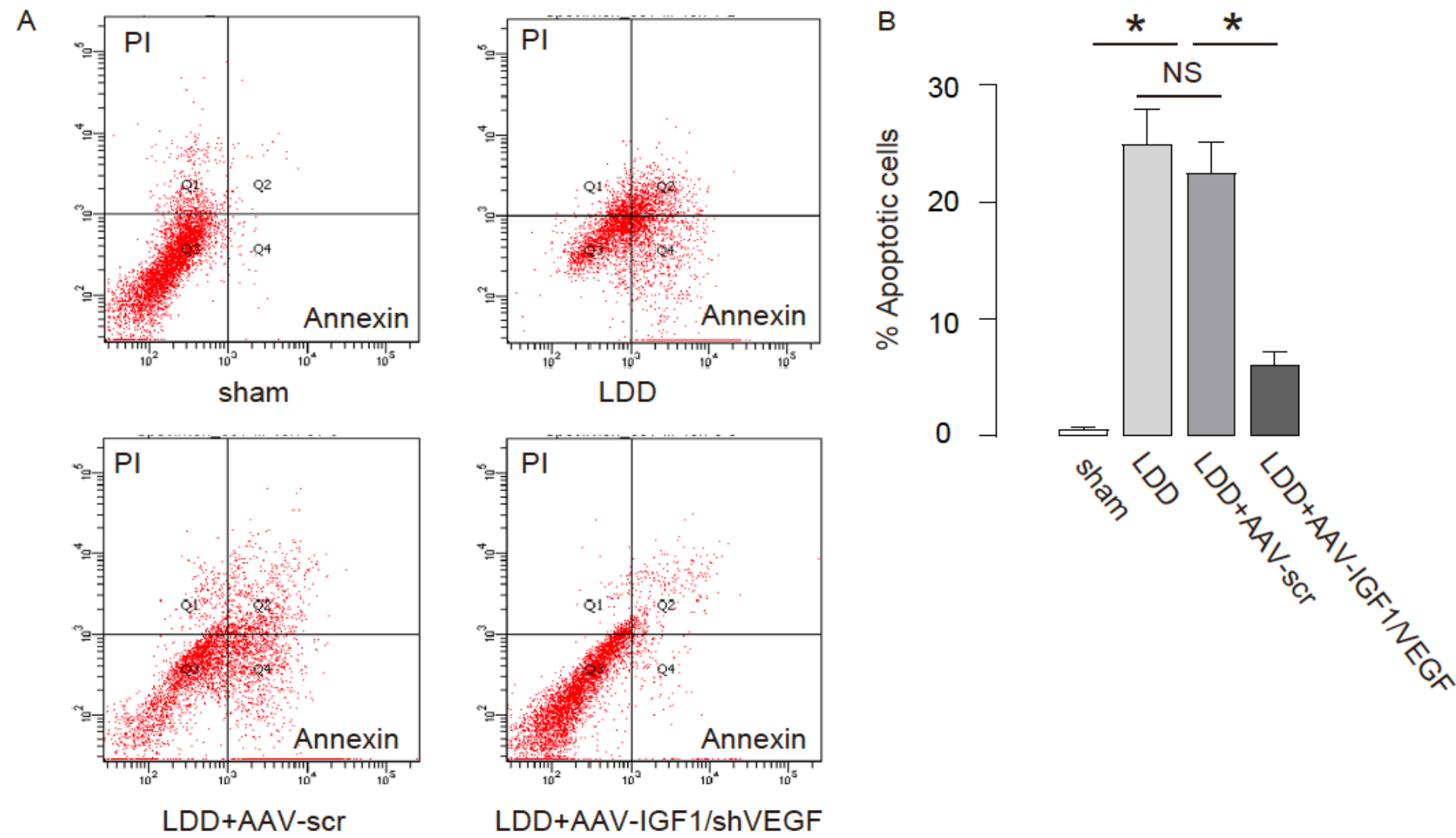

\section{Figure 5}

AAV-IGF1/shVEGF treatment reduces cell apoptosis in spine tissue after LDD (A-B) The disc cell apoptosis is assessed by examination of the dissociated single cells from the rat vertebral pulp and annulus fibrosus 8 weeks after treatments by flow-cytometry-based apoptosis assay, shown by representative flow plots $(A)$ and by quantification (B). ${ }^{*} p<0.05$. NS: non-significant. $N=10$. 

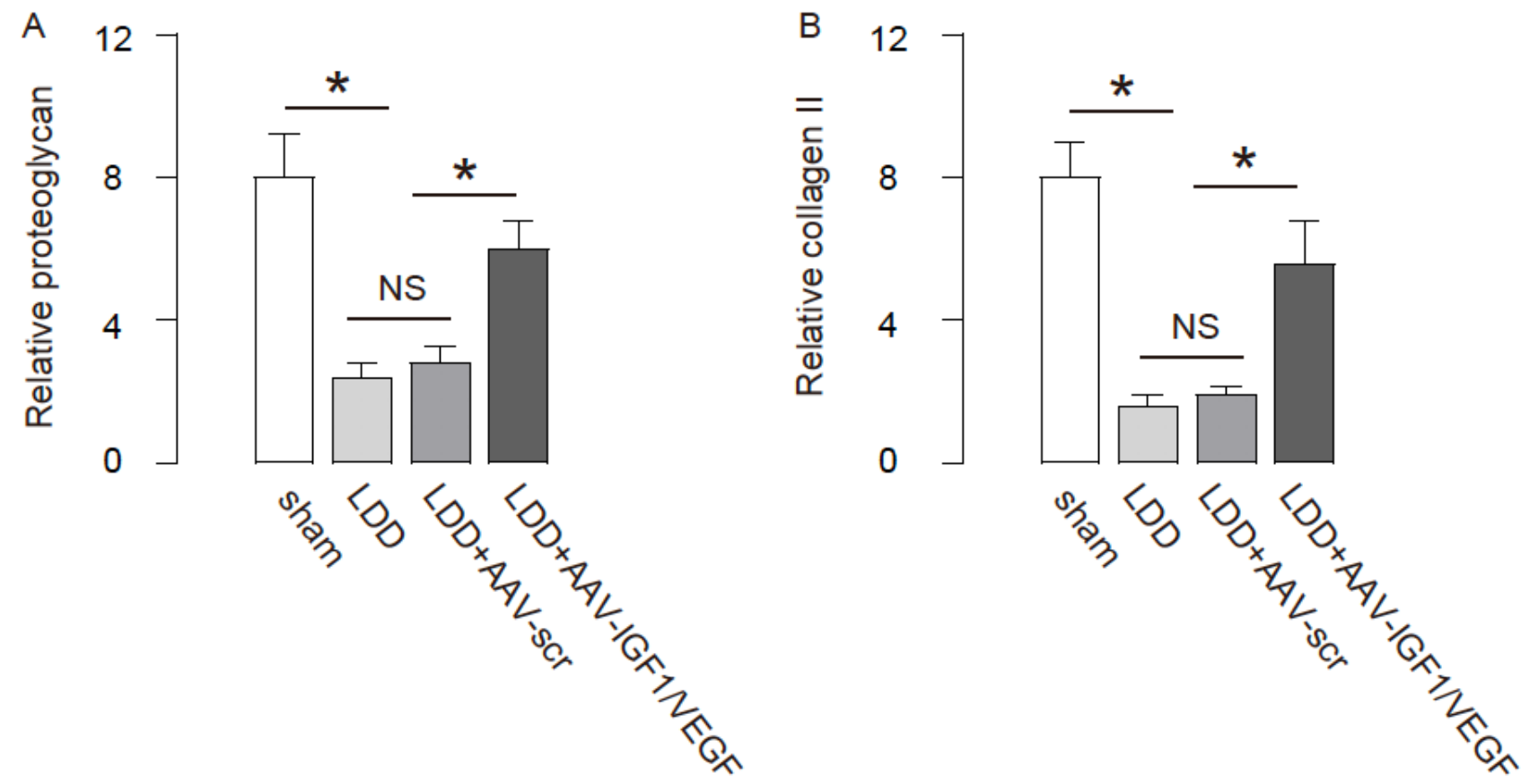

\section{Figure 6}

AAV-IGF1/shVEGF treatment enhances spine proteoglycan and collagen II after LDD (A-B) ELISA for the levels of proteoglycan (A) and collagen II (B) in the rat vertebral pulp and annulus fibrosus 8 weeks after treatments. ${ }^{*} \mathrm{p}<0.05$. NS: non-significant. $\mathrm{N}=10$. 